\title{
RADIO IMAGING OF SYMBIOTIC STARS
}

\author{
A.R. Taylor \\ Department of Physics, University of Calgary \\ Calgary, Alberta, Canada
}

\begin{abstract}
A brief review of radio observations of individual symbiotic stars is presented, with emphasis on radio imaging of the circumstellar nebulae. The ionized nebulae are catagorized into two types: outburst ejecta and stellar winds. Among the ejecta-type there is a strong tendency for bipolar or jet morphology. In the case of the quiescent, stellar wind emitter, a few very wide binary systems (eg. H1-36) offer the potential of directly viewing the effects of the binary interaction.
\end{abstract}

\section{INTRODUCTION}

The 1980's have seen major advances in radio investigations of symbiotic stars, primarily due to the combination of high sensitivity and resolving power provided by the NRAO Very Large Array. The results of an extensive, multi-frequency, VLA survey of symbiotic stars are reviewed by Seaquist elsewhere in this volume. A further area of radio investigation, that has lead to significant insights into the symbiotic phenomenon, is imaging at high resolution, to attempt to map the circumstellar ionized nebulae. This paper presents a brief review of observations of the radio structure of symbiotic stars, along with some new results.

\section{OBSERVATIONS OF RADIO STRUCTURE}

It is instructive to carry out a simple calculation that illustrates the limitations of current instrumentation for imaging of circumstellar gas. In the case of an optically-thick nebula with brightness temperature $\mathrm{T}_{B}=10^{4} \mathrm{~K}$ (the approximate equalibrium temperature for a photoionized gas), the radio flux density and angular size are related via

$$
S_{\nu}=5.7 \nu^{2} \theta^{2} \quad m J y,
$$

where $\nu$ is the observing frequency in $\mathrm{GHz}$ and $\theta$ is the diameter of either the source or the telescope beam, whichever is smaller. For symbiotic stars we are interested in regions with dimensions comparable to the binary separation, which 
ranges from a few $\mathrm{AU}$ for the short period systems (periods of a few years) to perhaps $1000 \mathrm{AU}$ for the very wide systems. A linear dimension of $100 \mathrm{AU}$ at a distance of $1 \mathrm{kpc}$ subtends an angle of 0.1 ", roughly equal to the resolving power of the VLA at $\lambda=2 \mathrm{~cm}$ in the highest resolution configuration. From equation (1) the flux density per beam area is then $\sim 10 \mathrm{mJy}$. Therefore a sensitivity of better than $1 \mathrm{mJy}$ is needed to successfully map emission at $10^{4} \mathrm{~K}$ on this scale. Note that the required sensitivity increases as the square of the angular resolution. To map emission with a resolution of 0.01 " (10 $\mathrm{AU}$ at $1 \mathrm{kpc}$ ) would require sensitivity of $.01 \mathrm{mJy}$. This combination of resolution and sensitivity is not yet available.

A corrallory of the above calculation is that optically-thick sources with flux density less than a few m.Jy will have angular sizes less than 0.1 " and are not mappable with the present generation of radio telescopes. Unfortunately, this is the case for the majority of radio detected symbiotic stars which are quiescent emitters at flux densities of typically $1-2$ mJy (Seaquist et al. 1984, Seaquist 1988 this volume). However, a few notable exceptions exist. Table 1 lists those symbiotic stars in which the structure of the nebula has been resolved in the radio. The list includes 10 stars. Of these, three, V1016 Cyg, HM Sge and AG Peg are symbiotic novae (see Allen 1980) one, RS Oph, is a recurrent nova and one, $\mathrm{CH}$ Cyg, has exhibited outburst behaviour similar to that of novae. This predominance of outburst-type stars in the list is a selection effect, since it is primarily these objects that obtain high enough flux densities to be mappable.

In table 1 the circumstellar nebulae have been catagorized into two types; ejecta and stellar winds. This is an attempt to distinguish between nebulae produced by an impulsive, energetic outburst and those resulting from a steady-state or, perhaps, slowly varying, outflow. Nebulae have been classed as ejecta if they have a clumpy appearance and can be associated with a known outburst of the system. Wind-type nebulae have a smooth, featureless morphology and are signified by an angular size that decreases with frequency. In the idealized case of an isothermal, uniform outflow $\theta \sim \nu^{(1.7}$ (see, for example, Wright and Barlow 1975). More generally, Seaquist and Taylor (1987) have shown that for a power law density distribution of the form $\rho \times r^{-p}$ and a temperature gradient given by $\mathrm{T} \propto r^{-n}$, the apparent angular size of the nebulae (ie. the radius corresponding to some value of the optical depth) will vary as $\theta \propto \nu^{-m}$ where

$$
m=\frac{2.1}{(2 p-1-1.35 n)}
$$

There is too little space here to discuss all of the stars in table 1 in detail, moreover, excellent discussions of a number are given elsewhere in this volume. Instead, some general comments and results are given below.

\subsection{Ejecta}

Those stars in table 1 that have undergone a recorded outburst are by far the best studied. The symbiotic novae are distinguished by the long time scale for evolution of the radio outburst relative to classical novae (eg. decades rather than years). The slow evolution is reflected in the expansion rate of the ejecta. 
Table 1. Symbiotic Stars Resolved at Radio

\begin{tabular}{|c|c|c|c|c|c|}
\hline Star & $\begin{array}{c}\text { Angular Size } \\
(")\end{array}$ & Type & $\begin{array}{c}\text { Radio } \\
\text { Variable? }\end{array}$ & Comment & Refs \\
\hline V1016 Cyg & 0.5 & ejecta + wind & $Y$ & symbiotic nova & 1 \\
\hline AG Peg & 1.5 & ejecta? & $\mathrm{Y}$ & symbiotic nova & 2,3 \\
\hline $\mathrm{R} A q \mathrm{r}$ & 6.4 & ejecta? & $\mathrm{N}$ & jet? & $4,5,6$ \\
\hline HM Sge & 0.5 & ejecta + wind & $\mathrm{Y}$ & symbiotic nova & 7 \\
\hline $\mathrm{CH}$ Cyg & 1.5 & ejecta & $\mathrm{Y}$ & jet & 8,9 \\
\hline RX Pup & 0.25 & wind & $\mathrm{Y}$ & & 10 \\
\hline $\mathrm{RS} O \mathrm{ph}$ & 0.2 & ejecta & $Y$ & recurrent nova & $11,12,13$ \\
\hline $\mathrm{H} 1-36$ & 5.0 & complex + wind & $\mathrm{N}$ & & 14 \\
\hline SS 96 & $0.17^{\times}$ & wind & $\mathrm{N}$ & & 14 \\
\hline Hen $1383^{\dagger}$ & 4.0 & ejecta? & $Y$ & jet? & 14 \\
\hline
\end{tabular}

\section{References}

1 Newell (1981)

3 Hjellming (1985)

5 Kafatos et al. (1983)

7 Kwok et al. (1984)

9 Taylor et al. this volume

11 Porcas et al. (1986)

13 Taylor et al. (1987)
2 Chigo and Cohen (1981)

4 Sopka et al. (1982)

6 Hollis et al. (1985)

8 Taylor et al. (1986)

10 Seaquist and Taylor (1987)

12 Hjellming et al. (1986)

14 This paper 
Figure 1 shows high resolution radio images at $\lambda=1.3 \mathrm{~cm}$ of the two recent symbiotic novae V1016 Cyg and HM Sge, with outbursts respectively in 1965 and 1975 . The morphology of the emission is very similar in the two cases; a central, bipolar source plus a more extended, low surface brightness halo. Lower frequency observations show that the extended emission has the characteristics of a stellar wind (eg. Kwok et al. 1984). The angular size of the bipolar features imply upper limits to the expansion velocity of $30 D_{k p c}$ $\mathrm{km}-\mathrm{s}^{-1}$ for V1016 Cyg and $50 D_{k p c}$ $\mathrm{km}-\mathrm{s}^{-1}$ for HM Sge, where $D_{k p c}$ is the distance in $\mathrm{kpc}$. A similar expansion velocity is implied for the third symbiotic nova AG Peg, which experienced an outburst around 1855 and currently has an angular size of 1.5 ". These low velocities are in marked contrast to the two other symbiotics with recorded radio outbursts, CH Cyg and RS Oph. Figure 2 shows radio images of $\mathrm{CH}$ Cyg at two epochs, illustrating the rapid expansion of the radio jet from this system. The expansion velocity for $D=400 \mathrm{pc}$ is $1050 / \sin i$

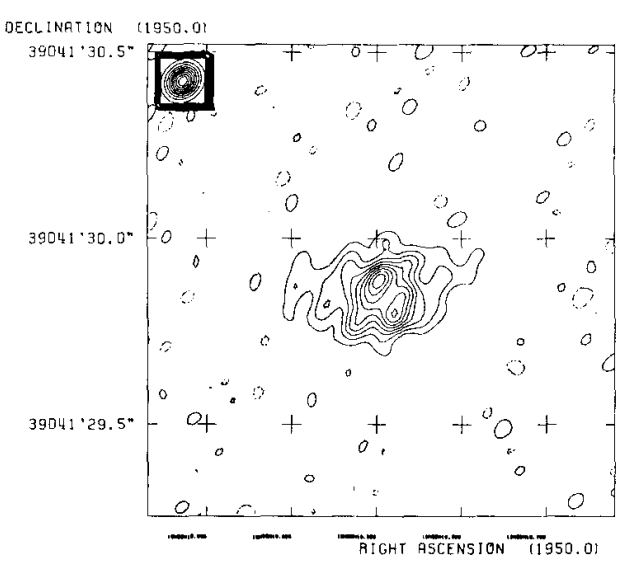

$\mathrm{km}-\mathrm{s}^{-1}$ (Taylor et al. 1986). A VLBI image of RS Oph indicates bipolar expansion of a mixture of free-free and non-thermal emitting gas at $\sim 3500 \mathrm{~km}-\mathrm{s}^{-1}$ (Porcas et al. 1987, Taylor et al. 1987).

The much lower nebular expansion velocities for the symbiotic novae can be explained by the interacting wind model of Kwok and Purton (1979), since in this case the region of high emission measure is a shell of shocked gas with predicted expansion velocity of $\sim 100 \mathrm{~km}^{-1}$ for reasonable parameters for the red giant and hot star winds. The lack of observable proper motion in the $\mathrm{R}$ Aqr jet (Hollis et al. 1985, Mauron et al. 1985) also indicate low outflow velocities for this star (less than a few $100 \mathrm{~km}-\mathrm{s}^{-1}$ ), although an interacting wind interpretation appears unlikely in this case. Solf and Ulrich (1985) have suggested that the R Aqr jet, and the larger scale nebulosity associated with the star, are the result of two, slowly expanding bipolar ejection events occuring 185 and 640 years ago. On the other hand, Kafatos et al. (1986) suggests that the jet components are produced 


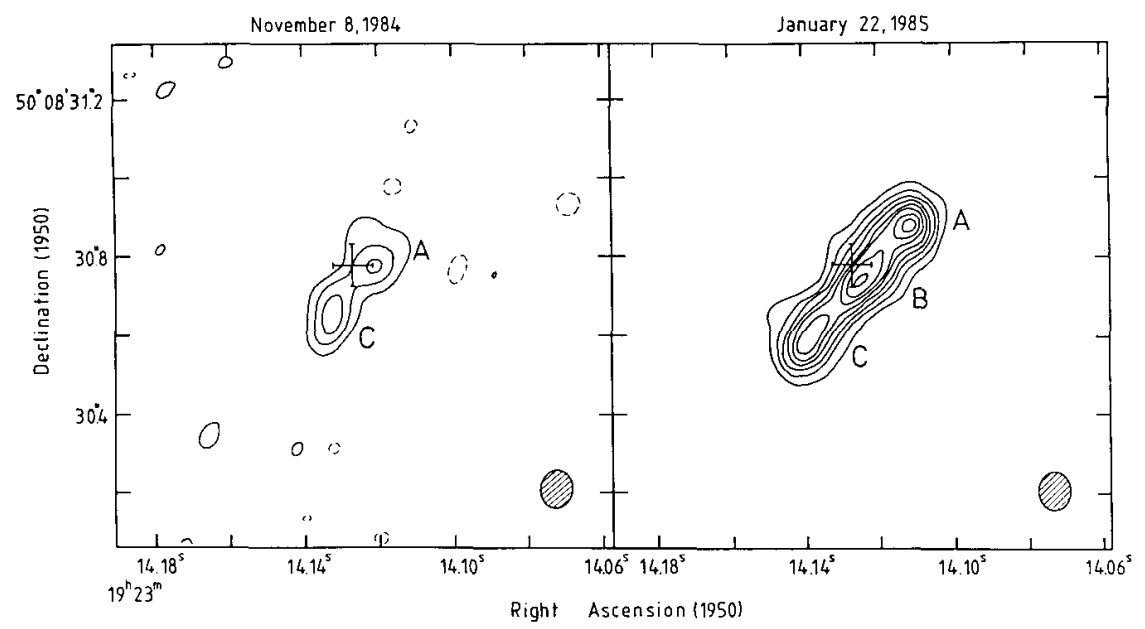

Figure 2. Two Radio Images of $\mathrm{CH}$ Cygni at $\lambda=2 \mathrm{~cm}$ separated by 75 day. The cross marks the position of the star. The angluar expansion rate of the jet is $1.1 " / \mathrm{yr}$.

by ejection of discrete parcels of material from a geometrically thick accretion disk at intervals of about 50 years.

While there does not appear to be a single ejection mechanism among the symbiotic systems that undergo radio outbursts, one element in common is the striking departure from spherical symmetry. In all cases the ejecta exhibit either bipolar or jet-like morphology. Such axial symmetry might reflect the presence of accretion disks or, particularly for the symbioitic novae, assymmetric mass loss from the red giant. The possibility that red giant winds are non-spherically symmetric is supported by recent MERLIN observations of the distribution of $\mathrm{OH}$ and $\mathrm{H}_{2} \mathrm{O}$ masers around red supergiants (eg. Chapman and Cohen 1986). Asymmetric mass loss has also been proposed by Manchester (1987) to account for the biannular appearance of many supernova renmants.

\subsection{Stellar Winds}

Seaquist et al. (1984), and Taylor and Seaquist (1984) proposed a binary model for quiescent radio emission from symbiotic stars in which the emission arises from free-free interaction in a portion of the red giant wind ionized by the hot companion. Among other things, this model accounts for the presence of a steady state, high-frequency turnover in the radio continuum spectra of some symbiotic stars. As pointed out by Allen (1983) such a turnover is the result of an inner boundary to the ionized nebula, leaving a cavity of neutral material around the red giant. A prediction of the model is that systems with large binary separation will tend to have a larger neutral region around the giant component, and will thus become optically-thin to free-free absorption at a lower frequency. If the binary separation were large enough, one might expect to observe the structure of the ionized cavity in the radio. While there is some curvature of the ra- 
dio spectra of symbiotic stars as a class in the $5-15 \mathrm{GHz}$ range (Seaquist, this volumme), most symbiotics are optically-thick up to the highest observable VLA frequency. Two notable exceptions are H1-36 and SS 96, which become opticallythin at $\sim 10 \mathrm{GHz}$. Model fits to the continuum spectra of these stars yield predicted values for the angular separation of the binary components of 0.5 " for H136 (Taylor and Seaquist 1984) and 0.4" for SS 96 (unpublished data).

Figure 3 shows a $\lambda=2 \mathrm{~cm}$ image of H1-36. The map shows a triple radio source with total extent roughly equal to the predicted angular separation of the binary components. Lower frequency observations with lower resolution revea] a more extended nebula with the characteristics of a stellar wind, consistent with the simple wind model. However, the picture on the scale of the binary separation is clearly much more complex. At the suggested separation of $\sim 10^{16} \mathrm{~cm}$, the red giant is well within its Roche lobe, and the lack of strong variability rules out an outburst/ejection event. The multi-component emission probably reflects the interaction of the hot component with the wind of the red giant. If the hot component is undergoing a nuclear shell flash, a high velocity wind may be present. The complex radio structure might then be a combination of shock-ionized and photo-ionized regions of the red giant wind. The weak eastern component might be chromospheric emission from the red giant itself, as has been observed recently in $\alpha$ Orjonis (Newell and Hjellming 1982) and $\alpha$ Sco (Hjellming and Newell 1983).

The radio image of SS 96 shows a single, assymetric, resolved source, without the complexity of the H1-36 image. Fits to the radio continuum spectrum also suggest a much lower luminosity for the hot component relative to H1-36.

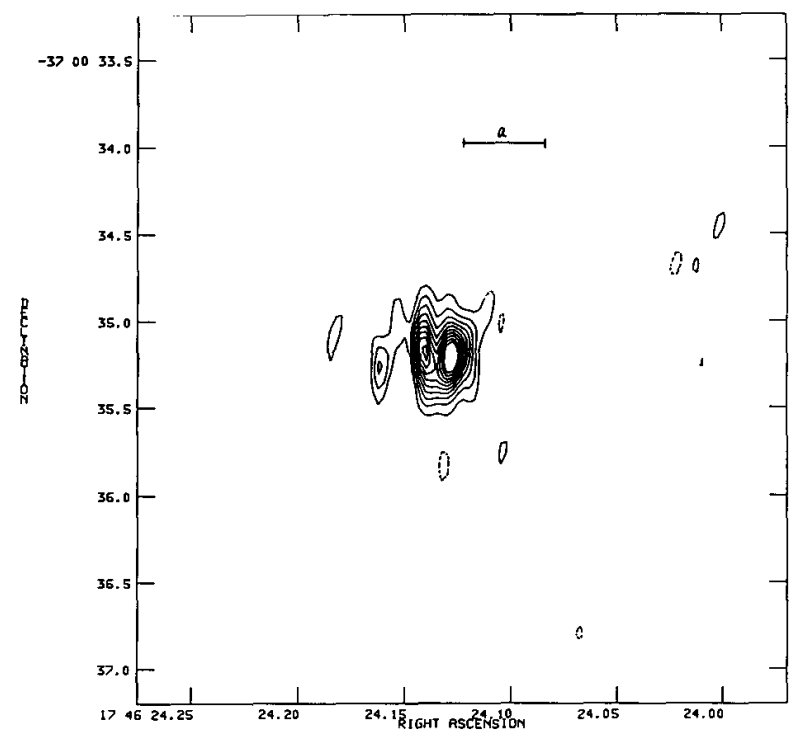

Figure 3. Radio Image of H1-36 at $\lambda=2 \mathrm{~cm}$. The angular dimension labeled $a$ is the predicted binary separation based on model fits to the radio continuum spectrum. 


\section{CONCLUSIONS}

Although radio imaging of the ionized nebulae of symbiotic stars is a rich and important area of investigation, it is a fairly recent endeavour. It has yet to benefit from a sytematic approach. For instance, second epoch images of many of the objects (eg. V1016Cyg, HM Sge and H1-36) remain to be obtained. The main limitation at this time, however, is still instrumental. Significant expansion of the list of radio resolved symbiotics must awajt instrumental developments. The completion of the Australia Telescope will add a number of southern stars to the list. Planned improvements to the sensitivity of MERLIN and the European VLBI network may allow another step in resolving power.

\section{References}

Allen, D.A. 1980, Mon. Not. Roy. astr. Soc., 192, 521.

Allen, D.A. 1983 Proc. Astr. Soc. Australia, 5, 211.

Chapman, J.M. and Cohen, R.J. 1986, Mon. Not. Roy. astr. Soc., 220, 513.

Chigo, F.D. and Cohen, N.L. 1981, Ap. J., 245, 988.

Hjellming, R.M. and Newell, R.T. 1983, Ap. J., 275, 704.

Hjellming, R.M. 1985 in Radio Stars, eds. R.M. Hjellming and D. Gibson, Dordrecht: Reidel, p. 97.

Hjellming, R.M., van Gorkum, J.H., Taylor, A.R., Seaquist, E.R., Padin, S., Davis, R.J. and Bode, M.F. 1986 Ap. J. Letters, 305, L71.

Hollis, J.M., Kafatos, M., Michalitsianos, A.G. and McAlister, H.A. 1985, Ap. J., 289,765 .

Kafatos, M., Hollis, J.M. and Michalitsianos, A.G. 1983, Ap. J. Letters, 267, $\mathrm{L} 103$.

Kafatos, M., Hollis, J.M. and Michalitsianos, A.G. 1986, Ap. J. Supp., 62, 853.

Kwok, S. and Purton, C.R. 1979, Ap. J., 229, 187.

Kwok, S., Bignell, R.C. and Purton. C.R. 1984, Ap. J., 279, 188.

Manchester, R.N. 1987, A. \& A., 171, 205.

Mauron, M., Nieto, J.L., Picat, J.P., Lelievre, G. and Sol, H. 1985, A. \& A. Letters, 142, L13.

Newell, R.T. 1981, Ph.D. Thesis, New Mexico Institute for Mining and Technology.

Newell, R.T. and Hjellming, R.M. Ap. J. Letters, 263, L85.

Porcas, R.W., Davis, R.J. and Graham, D.A. 1987, in RS Ophiuchi (1985) and the Recurrent Nova Phenomenon, ed. M.F. Bode, VNU Science Press:

Utrecht, p. 203.

Seaquist, E.R., Taylor, A.R. and Button, S. 1984 Ap. J., 284, 202.

Seaquist, E.R. and Taylor, A.R. 1987, Ap. J., 312, 813.

Solf, J. and Ulrich, H. 1985 A. \& A., 148, 274.

Sopka, R.J., Herbig, G., Kafatos, M. and Michalitsianos, A.G. 1982, Ap. J. Letters, 258, L35.

Taylor, A.R. and Seaquist, E.R. 1984, Ap. J., 286, 263.

Taylor, A.R., Seaquist, E.R. and Mattei 1986, Nature, 319. 38.

Taylor, A.R., Davis, R.J., Porcas, R.W. and Bode, M.F. 1987, Mon. Not. Roy. astr. Soc., submitted.

Wright, A.E. and Barlow, M.J. 1975, Mon. Not. Roy. astr. Soc., 170, 41. 